EPJ Web of Conferences 31, 00009 (2012)

DOI: $10.1051 /$ epjconf/20123100009

(C) Owned by the authors, published by EDP Sciences - SIF, 2012

\title{
Isospin and symmetry energy - From the laboratory to compact stars
}

\author{
Y. LEIFELS for the FOPI and ASYEOS Collaborations
}

GSI Helmholtz-Center for Heavy Ion Research Planck Str. 1, D-64921 Darmstadt, Germany

\begin{abstract}
The equation of state of asymmetric nuclear matter is still controversial, as predictions at subsaturation as well as above-normal density diverge widely. Several experimental observables measured in heavyion collisions in the energy range 0.1-1.500 AGeV are discussed. Estimates of the density dependence of the symmetry energy are derived from comparison of experimental results with those of transport codes with different implementations of the potential part of the symmetry energy are presented.
\end{abstract}

\section{Introduction}

Understanding the equation of state (EOS) of nuclear matter is of fundamental importance in many areas of nuclear physics and astrophysics [1]. The nuclear EOS describes the relation between density, pressure, energy, temperature and the asymmetry $\delta=\left(\rho_{n}-\rho_{p}\right) / \rho$, where $\rho_{n}, \rho_{n}$, and $\rho$ are neutron, proton and nuclear matter densities, respectively. The EOS may be divided into a symmetric matter part independent of the isospin asymmetry and an isospin term, also quoted as symmetry energy $E_{\text {sym }}(\rho)$, that enters with a factor $\delta^{2}$ into the equation of state [2].

Knowledge on the nuclear symmetry energy $E_{\text {sym }}(\rho)$ is critical in many aspects: At densities at and lower than saturation density $\rho_{0}$, symmetry energy influences nuclear masses, neutron skins, pygmy resonance, and nuclear structure at the drip line. In heavy ion collisions around the Fermi energy 
it governs the competition between dissipative mechanisms, and manifests itself through neutron distillation in fragmentation, which is a signature of a phase transition. At higher incident beam energies and nuclear matter densities larger $\rho_{0}$ the symmetry energy affects nucleon/cluster emission, collective flows and meson production, and at very high baryon densities a large symmetry repulsion may lead to an "earlier" hadron-deconfinement transition in neutron rich matter [3]. The EOS of asymmetric nuclear matter is also a quantity of crucial significance in understanding the physics of isolated and binary neutron stars, type II supernovae and neutron star mergers. Experimental information about the EOS can help to provide improved predictions for neutron star observables such as stellar radii and moments of inertia [1], crustal vibration frequencies, and neutron star cooling rates, which are currently being investigated with ground-based and satellite observatories.

There are different means to study the symmetry energy of the nuclear matter equation of state, but heavy ion collisions provide the only way to explore densities different from the saturation density $\rho_{0}$ in the laboratory, as in the course of such a collision matter suffers compression and expansion phases. Several observables have been proposed by theoreticians (for reviews, see $[1,3]$ ) to be sensitive to the symmetry energy and the experimental search for measurable effects started some years ago with stable-isotope beams and targets spanning the largest possible $\mathrm{N} / \mathrm{Z}$ range.

Those investigations can be categorized into three approaches:

- The N/Z of target and/or projectile is varied along isotopic chains; the system mass is changing, but not its charge.

- The N/Z of target and/or projectile is varied at constant mass: the system charge is changed keeping its mass stable.

- The system is unchanged, but isospin pairs serve as probes.

The prediction of the symmetry energy $E_{\text {sym }}(\rho)$ which can be derived from the experiments rely on models, generally microscopic transport codes simulating nuclear collisions, or sometimes statistical models which are claimed to give the absolute value of the symmetry energy under given density and temperature conditions.

\section{Symmetry energy}

The key ingredient for constructing the nuclear matter equation of state is the basic nucleon-nucleon interaction. Until now our understanding on 

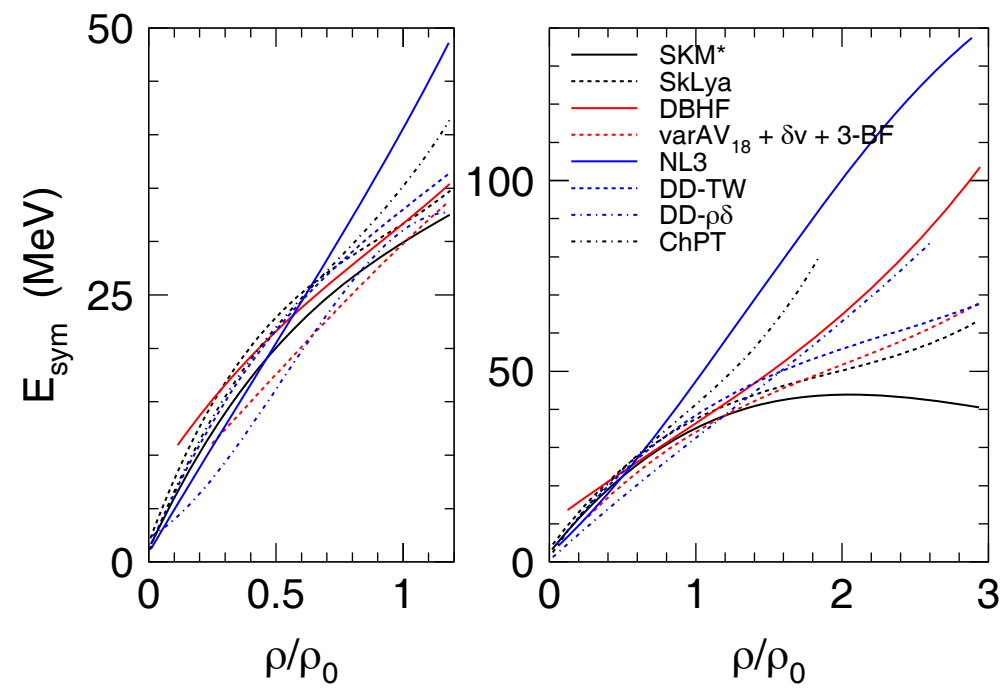

Figure 1: (Color Online) Symmetry energy as a function of density as calculated with different theoretical models. The left panel shows the low density region while the right panel displays the high density range. Figure is taken from [2].

nucleon-nucleon interactions derives from studying rather symmetric nuclear matter at moderate densities on the one hand and massive neutron rich astrophysical objects on the other. Bridging this gap in accordance with known realistic features of nuclear and hadronic physics is a major challenge for theoreticians and experimentalists.

Microscopic calculations of the energy functional of nuclear matter employing different approaches to the nucleon-nucleon interaction predict different forms of the EOS. As a result, the symmetry energy, which is the difference in energy between pure neutron and symmetric matter, shows very different behaviors. Fig. 1 (taken from [2]) compares the symmetry energy $E_{\text {sym }}(\rho)$ predicted by various calculations using different approaches, including energy functionals from Dirac-Brückner-Hartree-Fock calculations (DBHF), Relativistic Mean Field (DD-TW and DD- $\rho \delta$ ), and two Skyrme predictions $\left(\mathrm{SKM}^{*}\right.$ and SkLya). The left panel zooms the low-density region, while the right panel shows the high density behavior of $E_{\text {sym }}$. Most models coincide at or slightly below normal nuclear matter density, which demonstrates that constraints from finite nuclei are active for an average density. However, the extrapolations to supra-normal densities diverge dramatically. More recent calculations show in addition a strong enhancement of the symmetry energy when 3-Body-Forces are included [3].

The different density dependences of $E_{\text {sym }}(\rho)$ can be described more 


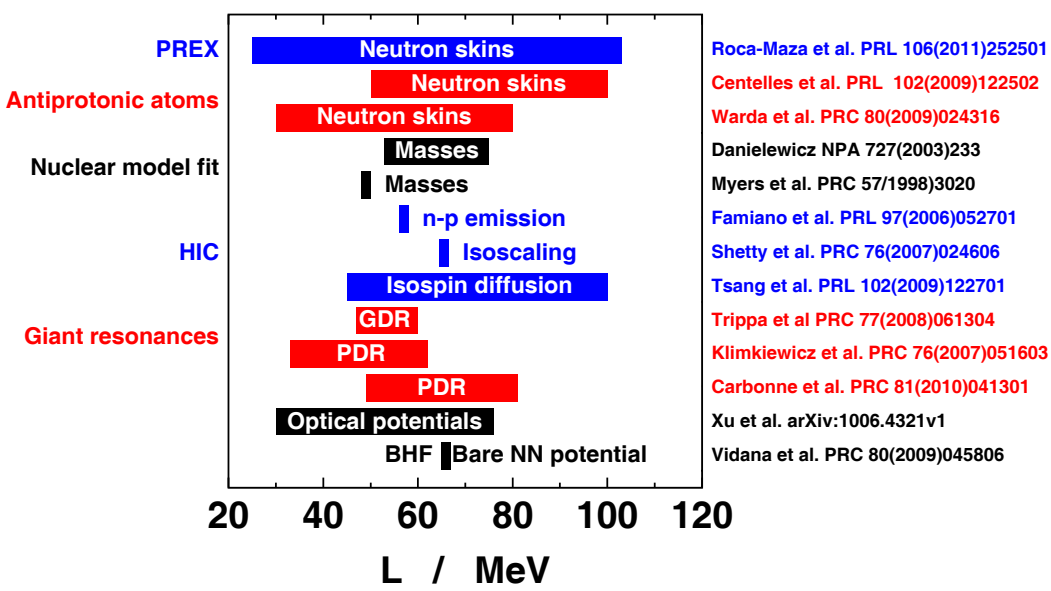

Figure 2: Compilation of the slope parameter $L$ determined by various methods at or below normal nuclear matter density $\rho_{0}$ (taken partially from $[6]$ ).

quantitatively by expanding the symmetry energy in terms of $\left(\rho-\rho_{0}\right) / \rho_{0}$ using the value of $E_{\text {sym }}\left(\rho=\rho_{0}\right)$ and the slope parameter $L\left(\rho_{0}\right)=$ $\left.3 \rho_{0} \frac{\delta E_{s y m}(\rho)}{\delta \rho}\right|_{\rho=\rho_{0}}$ leading to the following equation:

$$
E_{\text {sym }}(\rho)=E_{\text {sym }, 0}+\frac{L\left(\rho_{0}\right)}{3}\left(\frac{\left(\rho-\rho_{0}\right)}{\rho_{0}}\right)+\frac{K_{\text {sym }}}{18}\left(\frac{\left(\rho-\rho_{0}\right)}{\rho_{0}}\right)^{2}+\ldots
$$

where $K_{\text {sym }}$ is referred to as curvature parameter. If $L\left(\rho_{0}\right)$ is large $(L>$ $100 \mathrm{MeV}$ ) the symmetry energy increases strongly with density, ie. "stiff" dependence, if $L$ is small $(L<70 \mathrm{MeV})$ the increase of $E_{\text {sym }}(\rho)$ is only moderate with density and called "soft".

\section{Symmetry energy at low densities}

The value of $E_{\text {sym }}\left(\rho_{0}\right)$ is known to be around 25-34 MeV mainly from analyzing nuclear masses (e.g. [5]). The slope parameter $L\left(\rho_{0}\right)$ has been extensively studied but remains much more uncertain. Fig. 2 shows a compilation of values for the slope parameter $L\left(\rho_{0}\right)$ resulting from various experiments or theoretical calculations. These include the isospin diffusion, neutron/proton ratio of pre-equilibrium nucleon emission, giant dipole as well as pygmy dipole resonances, and neutron-skins of heavy nuclei studied in proton elastic scattering, in anti-protonic atoms, and more recently by parity violating electron scattering (quoted PREX [6]). The thickness of 
neutron skins $\Delta r_{n p}=\sqrt{\left\langle r_{n}^{2}\right\rangle}-\sqrt{\left\langle r_{p}^{2}\right\rangle}$ depends linearly not only on the slope parameter $L$ [7], but also on $E_{\text {sym }}\left(\rho_{0}\right)$ and the curvature parameter $K$.

Heavy ion reactions at moderate beam energies of nuclei with varying $\mathrm{N} / \mathrm{Z}$ are a sensitive tool to study the density dependence of the symmetry energy at sub-normal nuclear matter densities. Isospin diffusion in semiperipheral collisions may serve as an example. It is caused by an isospin gradient between the projectile and the target and leads to an exchange of neutrons and protons across the neck region in a semi-peripheral collision. The symmetry energy governs the pace of this equilibration process: if the symmetry energy has a stiff density dependence the degree of isospin diffusion will be small, if it is soft diffusion is large and equilibration is reached rapidly. Experimentally the $\mathrm{N} / \mathrm{Z}$ contents of the expanding system can be deduced from isobaric yield ratios (e.g. ${ }^{7} \mathrm{Li} /{ }^{7} \mathrm{Be}$ ) measured as a function of rapidity [8], these experimental results are compared with the predictions of transport models to obtain an estimate for the slope parameter $L$ (compare Fig. 2.

The values of $L$ compiled in Fig. 2 scatter between 30 and $100 \mathrm{MeV}$. The average slope parameter one may extract as a mean value of all these studies is $L \approx 60 \pm 10 \mathrm{MeV}$, which is equivalent to a rather "soft" dependence of $E_{\text {sym }}$ on density.

\section{Symmetry energy at high densities}

In contrast to the situation at and below normal nuclear matter density, very few constraints exist on the symmetry energy at supra-normal densities, i.e. $\rho \approx 2 \rho_{0}$. Several potentially useful observables which should be sensitive to the symmetry energy at high densities have been identified:

Kaon production in heavy ion reactions at energies close to the production threshold in NN collisions $\left(E_{t h r, N N}=1.6 \mathrm{GeV}\right)$ has proven to be sensitive to the densities reached in the course of the collision. In particular, kaon yields are a sensitive and robust observable to constrain the nuclear EOS. Thus the isospin contents of the produced strange mesons is claimed to provide information on the symmetry energy at high densities. The FOPI collaboration investigated $\mathrm{K}^{0}$ and $\mathrm{K}^{+}$production in the ${ }^{96} \mathrm{Ru}+\mathrm{Ru}$ and ${ }^{96} \mathrm{Zr}+\mathrm{Zr}$ systems. These systems have the same mass but different isospin. The results together with various model predictions are shown in Fig. 3 (left side). Obviously, the experimental data of the isotopic double ratio for kaons is described by the model predictions but the variation of these 

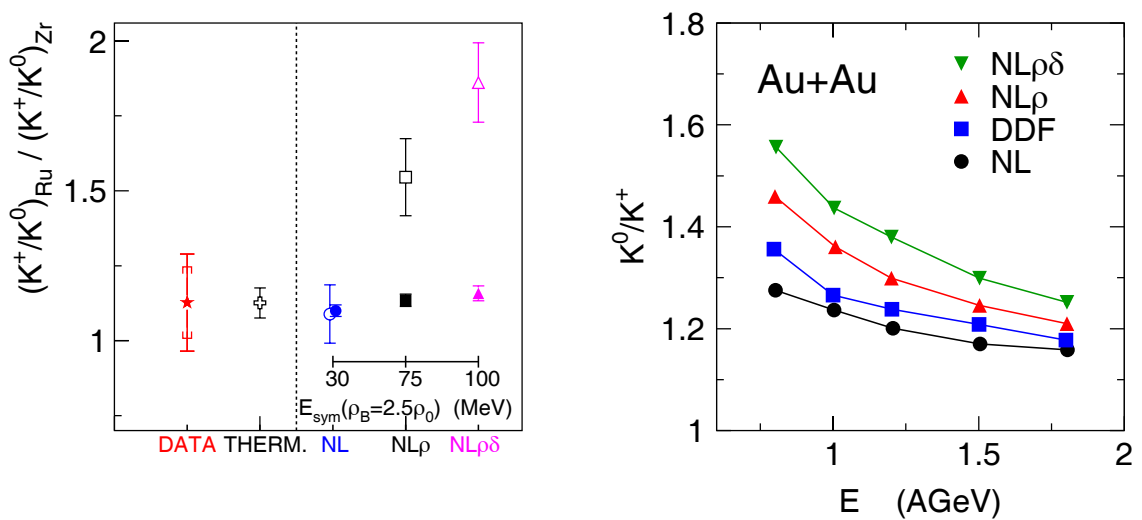

Figure 3: Left panel: Experimental ratio $\left(\mathrm{K}^{+} / \mathrm{K}^{0}\right)_{\mathrm{Ru}} /\left(\mathrm{K}^{+} / \mathrm{K}^{0}\right)_{\mathrm{Zr}}$ (stars) and predictions of a thermal model code and a BUU transport model employing three different approaches for the symmetry energy, NL, NL $\rho$ and NL $\rho \delta$ with increasing stiffness, and two different scenarios, i.e. INM (infinite nuclear matter, open symbols) and HIC (heavy ion collision, filled symbols). Right panel: $\mathrm{K}^{0} / \mathrm{K}^{+}$ratio as a function of incident energy for $\mathrm{Au}+\mathrm{Au}$ collisions calculated for different assumptions on the density dependence of the symmetry energy.

predictions with the magnitude of $E_{\text {sym }}(\rho)$ is only very small. In contrast to the model results for a heavy ion reaction scenario there is a significant variation of the kaon ratio with the behavior of $E_{\text {sym }}(\rho)$ in an infinite nuclear matter calculation. This finding corroborates the assumption that the isotopic kaon ratio might be a sensitive observable in heavy ion reactions. Indeed it can be shown that the sensitivity of the kaon ratio to $E_{\text {sym }}(\rho)$ is increasing with decreasing beam energy [3]. At energies substantially below the kaon production threshold in elementary collisions the sensitivity of the $K^{0} / K^{+}$ratio is enhanced as can be seen in Fig. 3 (right panel) where the evolution of the isotopic kaon ratio as a function of beam energy is shown for different assumptions on the symmetry energy. The ratio $K^{0} / K^{+}$is getting larger with the stiffness of $E_{\text {sym }}(\rho)$.

Also the isotopic yield ratio of pions (i.e. $\pi^{-} / \pi^{+}$) produced in heavy ion reactions is also strongly depending on the $\mathrm{N} / \mathrm{Z}$ of the colliding system. This effect was measured the first time at the LBL in Berkeley and confirmed recently by the FOPI collaboration: Pion production in symmetric collisions systems at incident energies ranging from 0.4 to $1.5 \mathrm{AGeV}$ have been measured. At high beam energies the $\pi^{-} / \pi^{+}$exhibit a linear (N/Z) dependence which is changing to a $(\mathrm{N} / \mathrm{Z})^{2}$ at the lowest beam energies investigated, see Fig. 4. 

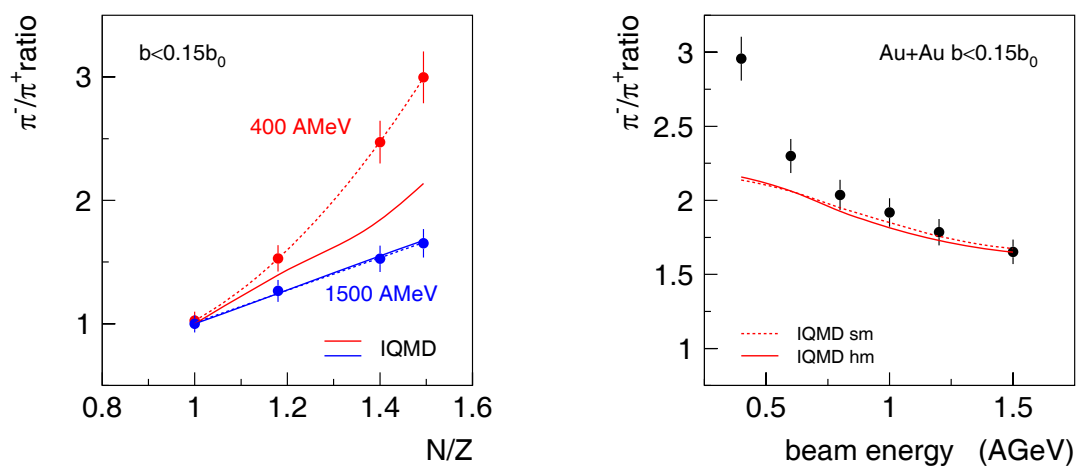

Figure 4: Left panel: $\pi^{-} / \pi^{+}$for $\mathrm{Au}+\mathrm{Au}$ collisions as a function of $\mathrm{N} / \mathrm{Z}$ for two different beam energies from [9]. The dashed lines are polynomial fits to the data (polynomials of first order at $1500 \mathrm{AMeV}$ and second order at $400 \mathrm{AMeV}$ ). The full lines are predictions from the IQMD transport code [10]. Right panel: $\pi^{-} / \pi^{+}$ for $\mathrm{Au}+\mathrm{Au}$ collisions as a function of beam energy. The dashed and full lines are IQMD predictions using a soft (dashed line) or stiff (full line) symmetric EOS.

Within an isospin and momentum dependent transport approach it was claimed that an agreement with these data is obtained when a very soft $E_{\text {sym }}(\rho)$ is employed [11], another transport calculation is reaching the opposite conclusion [12]. The possible origin of these discrepancies are discussed in [3], and may be connected with threshold effects in the pion production and the momentum dependence of self energy of the relevant particles (n, p, $\Delta$ etc.). The authors emphasize that it is important to measure neutron and proton emission together with the pion production channel to gain control on those effects.

Neutron and proton emission has been measured by a combination of the LAND neutron detector with a part of the FOPI set-up [13]. The LAND detector allows for the detection of neutrons and light charged particles simultaneously, and FOPI was utilized to reconstruct the reaction plane and the impact parameter to investigate directed and elliptic flow of neutrons. In Fig. 5 results of this experiment are shown for the ratio of differential elliptic flow parameters $v_{2}$ as a function of transverse momentum for semicentral $\mathrm{Au}+\mathrm{Au}$ collisions and compared with predictions of the UrQMD transport model [14]. The errors bars of the experimental data are quite large and mainly of statistical origin. The slope parameter $L\left(\rho_{0}\right)$ which is obtained from the comparison between data and model predictions amounts to $L=86 \pm 15 \mathrm{MeV}$. Although the error is quite large, a particular soft or a very stiff density dependence of the symmetry energy is ruled out. 


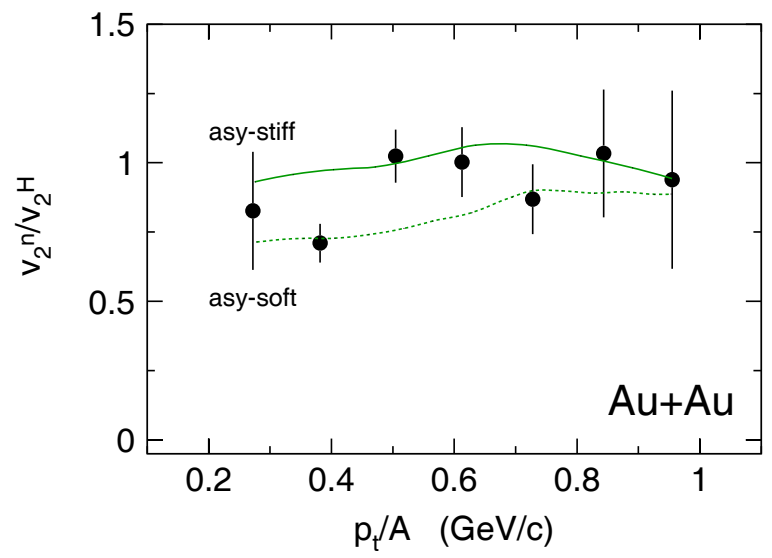

Figure 5: Ratio of the elliptic flow parameters $v_{2}$ of neutrons and hydrogens isotopes for moderately central $\left(\mathrm{b}<7.5 \mathrm{fm}\right.$ ) collisions of ${ }^{197} \mathrm{Au}+{ }^{197} \mathrm{Au}$ at $400 \mathrm{AMeV}$, integrated within the rapidity interval $0.25>y / y_{p}>0.75$, as a function of the transverse momentum per nucleon $p_{t} / \mathrm{A}$ from [14]. The symbols represent the experimental data. The UrQMD predictions for a "stiff" and a "soft" $E_{\text {sym }}(\rho)$ are given by the full and dashed lines, respectively.

Those findings have been confirmed recently by using a different transport code [15]. Nevertheless, the statistical significance of the data is rather insufficient and there was an attempt to remeasure neutron/proton flow in $\mathrm{Au}+\mathrm{Au}$ collisions at an incident energy $E_{\text {beam }}=400 \mathrm{AMeV}$ by the ASYEOS collaboration [16].

Another observable predicted to be sensitive to $E_{\text {sym }}(\rho)$ is the directed and elliptic flow of $\mathrm{t}$ and ${ }^{3} \mathrm{He}[1]$. Both have been measured by the FOPI collaboration for $\mathrm{Au}+\mathrm{Au}$ collisions between 0.4 and $1.5 \mathrm{AGeV}$ [17]. Elliptic flow data as a function of normalized rapidty for $\mathrm{t}$ and ${ }^{3} \mathrm{He}$ at two different incident energies are shown in Fig. 6. At low energies elliptic flows of $t$ and ${ }^{3} \mathrm{He}$ are nearly equal but they develop differences at higher energies. The elliptic flow value $v_{2}$ of $t$ is larger at mid-rapidity and when plotted as a function of rapidity it develops a bell shaped form which is more compact than the one of ${ }^{3} \mathrm{He}$. UrQMD predictions are on average describing the measured data well, but the model calculations predict nearly the same elliptic flow for $\mathrm{t}$ and ${ }^{3} \mathrm{He}$ independent on the beam energy and - more over - independent on $E_{\text {sym }}(\rho)$. Obviously, the symmetry energy is not the only factor which influences the flow patterns of isotopic partners emitted in heavy ion reactions. More systematic comparisons to state-of-the-art model calculations and experimental data are needed to elucidate the different contributions. 

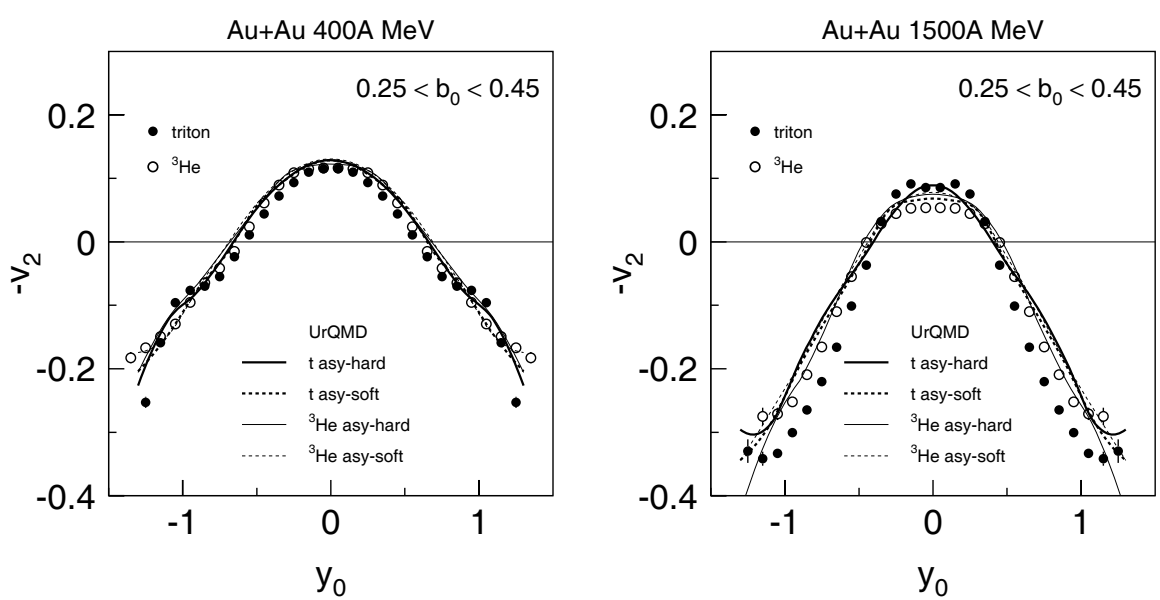

Figure 6: Left panel: Elliptic flow $-v_{2}$ of $\mathrm{t}$ and ${ }^{3} \mathrm{He}$ as a function of normalized rapidity $y_{0}=\frac{y_{l a b}}{y_{c m s}}-1$. for $\mathrm{Au}+\mathrm{Au}$ collisions at $400 \mathrm{AMeV}$ at mid-central collisions [17] together with the results of UrQMD for two different $E_{\text {sym }}(\rho)$ [14]. Right panel: Same but for $E_{\text {beam }}=1500 \mathrm{AMeV}$.

\section{Summary and conclusions}

Symmetry effects are very small, because the contribution of the symmetry energy enters with a factor $\delta^{2}$ into the equation of the state of nuclear matter. Since $\delta$ is rather small even for the most neutron rich stable isotopes it is evident that those studies have to be extended to radioactive beams with enhanced neutron-proton asymmetry. Experimental facilities, e.g. MSU/FRIB, GANIL/SPIRAL2, and FAIR/SFRS, will become available in the near future, which allow further studies of the symmetry energy by different experimental methods.

Very few experimental constraints exist on the symmetry energy at supra-saturation densities and above. This is the domain with the greatest theoretical uncertainties and the largest impact on the understanding of neutron stars. The behavior of the symmetry energy at supra-saturation densities can only be explored in terrestrial laboratories by using relativistic heavy ion collisions of isospin asymmetric nuclei. Several potentially useful experimental observables which are - according to models - sensitive to the behavior of the symmetry energy at supra-saturation densities have been identified. It is likely that consistent measurements of all of these will be necessary to provide definitive constraints on the symmetry energy. 
Acknowledgments: This work was supported by the German BMBF under Contract No. 06HD190I, by the Korea Science and Engineering Foundation (KOSEF) under Grant No. F01-2006- 000-10035-0, by the mutual agreement between GSI and IN2P3/CEA, by the Hungarian OTKA under Grant No. 47168, by DFG (Projekt 446-KOR-113/76/04) and by the EU, 6th Framework Program, Integrated Infrastructure: Strongly Interacting Matter (Hadron Physics), Contract No. RII3-CT-2004-506078.

\section{References}

[1] B. A. Li, L. W. Chen and C. M. Ko, Phys. Rept. 464 (2008) 113

[2] C. Fuchs and H. H. Wolter, Eur. Phys. J. A 30 (2006) 5

[3] M. Di Toro et al., J. Phys. G 37 (2010) 083101

[4] D. V. Shetty et al., Phys. Rev. C 75 (2007) 034602

[5] P. Danielewicz, Nucl. Phys. A 727 (2003) 233

[6] X. Roca-Maza et al., Phys. Rev. Lett. 106 (2011) 252501

[7] S. Typel, B.A. Brown, Phys. Rev. C 64 (2001) 027302

[8] M. B. Tsang et al., Phys. Rev. Lett. 92 (2004) 062701.

[9] W. Reisdorf et al. [FOPI collaboration], Nucl. Phys. A 781 (2007) 459

[10] C. Hartnack et al., Eur. Phys. J 151 (1996) 1.

[11] Z. Xiao et al., Phys. Rev. Lett. 102 (2009) 062502.

[12] Zhao-Qing Feng et al., Phys. Lett. 683 (2010) 140.

[13] Y. Leifels et al. [FOPI Collaboration], Phys. Rev. Lett. 71 (1993) 963.

[14] P. Russotto et al., Phys. Lett. B 697 (2011) 471

[15] M. Cozma et al., Phys. Lett. B 700 (2011) 139

[16] P. Russotto et al., contribution to this conference

[17] W. Reisdorf et al. [FOPI collaboration], Nucl. Phys. A 876 (2012) 1 